\title{
Cooperating school classes
}

\author{
Bente Evjemo \\ Arne Ketil Eidsvik \\ Thore Danielsen \\ Telenor Research \\ Tromsø \\ Norway
}

\begin{abstract}
This paper discusses a field trial to study the usability of videoconferencing in two elementary schools, one in Norway, the other in Sweden. Teachers of 5th grade classes were introduced to the communication medium and were told to look for cooperating tasks. The results indicate positive effects on the process of foreign language learning. In addition videoconferencing supplied an interesting meeting point between children in Norway and Sweden strengthening their sense of community both within each individual class and between the two classes. Finally new cooperation partners, new cooperation methods and new topics on which to focus, are discussed.
\end{abstract}

Main conference themes: distance learning

Educational areas: elementary education, primary education

Study topics:

Secondary keywords: collaborative learning, culture, pedagogy, teleconferencing 


\section{INTRODUCTION}

The videoconferencing service is often marketed as a substitute for real meetings between energetic business people. Videoconferences have also been tested for educational purposes usually with grown-up people as participants [1, 2] and in literature little or no reference is made to videoconferencing for younger users. We have therefore done a field trial aimed to study the usability of videoconferencing for children in their relationships with other children in primary school.

This field trial is one of several activities included in a project 'Adolescence and Education' which itself is part of a Norwegian Telecom Research's project 'Telecommunication within the Local Public Sector'.

\section{Friendship classes}

It is not unusual for school classes to have friendship classes somewhere else in the country, or even in a foreign country. We have exploited the relationship between 5th grade classes at Borgtun Primary School in Tromsoe, Norway, and Norrbyskolan Primary School in Hortlax, Sweden. It is a long way between Tromsoe and Hortlax - not just geographically and in actual travel time (the flight goes via Oslo and Stockholm) - but culturally as well. Hortlax is a small village outside Piteaa in the northern part of Sweden characterized by steady climate and rural peace. In comparison Tromsoe is a busy city with a dash of the transient coastal culture.

The kids have been for about one year in contact using normal mail as communication medium. Each pupil has a penpal in the other class. As a milestone the pupils have the prospect of a future visit. In the case reported here the pupils from Norrbyskolan will leave for Tromsoe and their friendship class on May 17th, the National Day in Norway.

\section{Just for fun?}

Writing letters is one way of communicating-videoconferencing is something totally different. The aim in both cases is to establish something outside the everyday school situation. The pupils find this amusing, but the teachers have-as usual-underlying motives:

- Children from the northern part of Scandinavia should be aware of each other and a feeling of togetherness and solidarity across the country borders should be developed. The way the children present themselves and their domestic situations is relevant in this context. These provide concrete examples of children's everyday situations. 
- Direct contact between Norwegian and Swedish children has advantages for mastering foreign languages. They are forced to express themselves clearly and distinctly, have to search for synonyms in their own language in order to be understood and pick up words and expressions from the other language.

- Finally fellow feeling among the pupils should be developed and a sense of community encouraged. This aspect was especially important for the Tromsoe teacher because several new pupils had joined her class at the beginning of the school year.

\section{THE WAY WE DID IT}

Borgtun Elementary School is situated about 5 minutes by bus from a studio in Tromsoe centre. The children in Piteaa have a ride of 15 minutes to a studio. The teachers and their respective pupils had full disposal of the $2 \mathrm{Mbit} / \mathrm{s}$ connection for $3 \times 2$ hours. Visits to the studio were within 14 days.

\section{Getting used to the idea of doing it: preparations}

Teachers had a free hand, but should look for cooperative tasks. Both teachers were interested in trying out new technology and new teaching methods. In collaboration they worked out a professional arrangement for the experiment. Most preparations were made during one meeting in person. Later corrections were made through facsimile and telephone. Three sessions were planned. The first was a kind of get-together to become familiar with each other. The second was a 'boasting' session where the pupils were supposed to tell about the excellence of their own school and city without restraint, The last session had a more professional character and aimed to compare results from an environmental experiment. We as researchers prepared by practising how to stay invisible during the conferencing.

\section{The way it happened}

There were 25 pupils in Tromsoe and 21 in Piteaa. The pupils were divided into three groups to ensure that all of them should get equal change to take part and that penpals would meet at the monitor. In Tromsoe, as in Piteaa, the teacher maintained control of the cameras, directed the troops in and out of the studio, chaired the program and did the necessary prompting.

Most of the time the camera pictures of the group, but was occasionally towards individual pupils. A still picture camera turned out to be suitable for showing details of pictures, maps and objects.

The teachers and the pupils had previously agreed on how to start the session. Each pupil had a separate message to bring which varied from session 
to session and depended on how well they were prepared, how much had been noted down and to what degree they kept to the manuscript. Most conversations went as scheduled. The first pupil from Tromsoe sees his Swedish penpal at the monitor and gives his message finishing off with a question to which the penpal can answer and comment. Then the teacher in Tromsoe introduces the next pupil. All sessions in the studio were taped for later study.

\section{COMMENTS}

The somewhat informal character of the experiment does not allow decisive conclusions as to the suitability of videconferencing in primary school. Our level of ambition was more on the suggestive side. We did however observe the children in action and summarized the experiences with both the children and the teachers.

\section{Comments from the pupils}

We talked to 43 of the total number of 46 children in groups of 3 and 4 and asked them the following questions:

- what was the most/least funny thing about videoconferencing?

- what was most/least difficult about videoconferencing?

- what was it like to see the children in the other class?

- what did you actually learn?

- do you think mum/dad understands what you have been doing?

"We were lucky to be chosen for this project!"- this was the conclusion of the Swedish children. Both the Swedish and the Norwegian pupils were most concerned by the fact that they could see the other class and talk to them directly. They specially mentioned the exciting meeting with their penpals: "fun to see them-they bustle about and are very lively", "they look different from what we thought" and "look forward to meeting them now we have seen them".

Listening to the language of a neighbouring country was fun as well and they were visibly proud being able to understand each other. The preparations within the class were mentioned as positive. The excitement of being down town was surely part of the event ("We even took part in a car crash and we played billiards at the hotel!"). No other class was invited-they were the selected children. 
They listed the difficult parts as:

- to deal with the delays;

- to talk into the microphone;

- to understand what the other party said;

- to keep quiet and wait.

Additionally they mentioned difficulties starting the conversation (lots of 'quiets', especially the first time)-partly due to delay problems caused by video compression. They felt nervous about speaking to so many people and it was sometimes hard to concentrate when those at the monitor were restless. Some of the Norwegian ones pointed out that they felt embarrassed making mistakes with their friends present. From the Swedish we got the opposite impression.

The children evaluated the first program to be the least successful one. They were nervous, they forgot what they were supposed to say and had the feeling of making mistakes all the time. The Swedes felt the Norwegians dominated the 'boast session', while the Norwegians were embarrassed by their own contribution to the third session on environmental issues.

By and by the children felt more confident in front of the camera, they dared to talk loudly and clearly, and got used to this somewhat special way of communicating. They saw the importance of being well prepared and the value of cooperating. Actually the long waiting time represented the greatest problem for the pupils. The Norwegians also mentioned the poor sound. One of them just longed both for bed and food, and found the whole thing miserable.

The pupils learned about each others districts (Tromsoe has a lot of tunnels and sports arenas-Piteaa has a jungle shop and miles of scooter tracks) and about each other. Their conclusion on environmental problems was described as: "There is more pollution in Sweden than in Norway". Also their parents understood what they had participated in: "They do know about a television set" the children said.

\section{Comments from the teachers}

The teachers were then asked the same questions, and the essentials from these interviews are quoted. For short we call them $\mathrm{N}$-teacher (Norwegian teacher) and S-teacher (Swedish teacher).

The expectations-were these fulfilled? The teachers stressed different aspects. S-teacher was specifically interested in the technical part. In addition she wanted the children to feel confident about their own behaviour and their own language. $\mathrm{N}$-teacher did not expect much of the technology, but hoped that the experiment would work out positively for the fellow feeling. Asked whether 
the expectations were fulfilled, S-teacher answered: "Yes, absolutely. The children have brought their experiences back into the classroom. They talk about voice production and compared themselves to the children in Tromsoe", and N-teacher: "Definitely yes, in spite of a great deal of work".

How did the studio function technically? Good for the Swedes, while the feedback from Norwegian side was "a somewhat poor sound".

How would you characterize the childrens' behaviour in the studio? S-teacher found that they were very excited the first time, but gradually became more able and relaxed. N-teacher found the first time almost too exciting. The last was more relaxed. The children brought pictures and drawings —on their own initiative - in order to better explain.

What consequences did the conferencing have for the sense of community and friendship to the other class? S-teacher: Great importance, they got a common experience. I can clearly see that the childrens' selfconfidence was strengthened. N-teacher: Yes. Really! Several pupils are now waiting for a penpal. I sense a positive development.

Did the trial stimulate the pupils to work harder? S-teacher: Yes, they have put more work into it. N-teacher: High professional level in Piteaa, so we had to reach out. Otherwise no special efforts. Generally I think it may have stimulated the professional interest.

Different effects on so-called bright and weak pupils? S-teacher: One girl who is a very quiet pupil, practically thrived. Some of the boys were more active and abler than expected as well. N-teacher: The bright ones grew brighter, but so did the weak ones as well. One weak and negatively oriented pupil suddenly wanted a penpal. Another one who can hardly read, did better. They get positive response for initiative and activities which are not usually part of the normal school day.

Are you left with negative impressions? S-teacher: More time consuming than presumed (travel to studio, for example)- the whole day was needed. The language barrier was stronger than expected. N-teacher: The preparations took a lot of time and the sessions came too close to each other. The waiting time outside the studio became too long.

Assuming that videoconferencing was available at school and the costs of no interest $\longrightarrow$ what would then be the main obstacle? S-teacher answered that she could see no practical problems. $\mathrm{N}$-teacher answered that available tools-books of exercises-would be necessary. Both teachers however stressed the unique possibility of learning the Nordic languages. 
Other comments? S-teacher: We have looked through the videos and discussed how it all functioned. We should train to be able to handle situations where language is an obstacle.

\section{DISCUSSION}

\section{What about the teachers' expectations?}

The teachers had hopes and beliefs in videoconferencing. We have no quantitative data on this, but base our evaluation on the teachers' assessments. They conclude that the expectations were fully met.

Solidarity past the borders: The pupils now know a lot more about each other's home towns and the ties between them grew stronger in a few hours. By gaining an insight in other childrens' everyday life, they will instinctively reflect on their own situation. This has also been noted in other tests in which communication tools have helped children get in touch with other languagecultural backgrounds [3]. The children particularly stressed that having seen and spoken directly to their penpal had been the best part of it.

In future they can now visualize their friends passing the red-painted 'jungle shop' on their way home to rabbits and younger sisters. Or they can see, with a dash of envy, their Swedish brothers speeding on the endless scooter tracks while the sun is shining. In the opposite direction, we may visualize the school yard of the Norwegian children with a view to high mountains and a giant bridge. The earth below is shaking and we can hear a boom. It comes from the digging work making the subterranean way connecting the Tromsoe island to the main land-an incredible thought for the inland people of Hortlax. This is a world different from what they know-this is the reality of their friends in Tromsoe.

Language training: The children pointed out the language as a funny element. At the same time language did cause some problems. This was an educationally very interesting situation: the situation forces the children to express themselves precisely. The ultimate alternative is to use the other party's words and phrases which felt natural at the next meeting.

Sense of community: From our spectator position we could clearly see the team effort involved: how have we done compared to the other class. However, other activities might equally have been used, e.g. making a newspaper, theatre, hikes, etc. We also noticed that the children gained more confidence in themselves. They experienced videoconferencing as a view into the grownups' world—a world which they otherwise only get glimpses of. 


\section{Technological and pedagogical issues}

Setup of the studio. The different setups of the studios in Tromsoe and Piteaa had an impact on the atmosphere in the studio and decided to what degree the children experienced the videoconferencing as a real meeting. In Piteaa the children had one large monitor to pay attention to. Outgoing pictures were shown in a corner of the large monitor in such a way that it did not attract much attention. We did notice more noise and less concentration in Tromsoe where the in- and outgoing pictures were not separated the same way. Nor did the setup of studio-arranged for 3-4 participants-render the best of conditions for a group of 8 active 11-year olds. It is important to prevent unnecessary disturbances when grown ups meet this way. For children it is an absolute demand. The same can be said about the quality of the sound. On the Norwegian side the sound was never good, and contributed to more noise than necessary. We also had a confirmation of what has previously been pointed out [2]; good sound is a must, while good pictures will render welcome positive effects.

Demand for pedagogical arrangement. Videoconferencing as a meeting place, information-channel and cooperation forum is special and demands special arrangements in order to give the customers good value for the money. In this test, the learning aspect was not distinctively stressed. Nevertheless our teachers wanted pedagogical guidelines and directions as well as educational material adapted for the situation in studio. This would further have contributed to the advantages of the medium, and at the same time reduced the extra work for the teachers, which in this context was disproportionally time-consuming. Moreover, we did observe that both the teachers and the pupils gradually became more able in using the possibilities of the medium. In order to make their messages easier they brought things to show, and sketched out drawings. The children focused more on the explanation and the direct response which made the dialogue spontaneous and intense. Afterwards, both teachers and pupils had ideas about further work.

Recordings were made of everything transmitted for the teachers and pupils to review the meetings in peace and quiet-allowing a total appraisal specifically of their own behaviour, choice of words and gesticulating. The feedback we have received has been positive to this possibility of review.

Previous studies suggest that so-called bright pupils strengthen their position compared to the weaker ones when technology is introduced. Experiences from our trial, however, suggest that the weaker pupils become more assertive. There is also research which suggests that the distance aspect of videoconferencing has positive aspects. This has been demonstrated in the therapeutic relations involving children [4] and might prove to be of interest in 
learning. Videoconferencing may not be a second-hand physical meeting place, but may have its own qualities.

\section{CONCLUSION}

This field trial points out the importance of arranging the studio in a way suitable for an educational or cooperative situation. In addition our field trial indicates - but does not give adequate data of - several positive aspects of videoconferencing in primary school of which the language training aspect must be stressed. The direct situation forces the pupils to face the language challenge and to use the other party's terms.

Further work could be started on a closer connection between children in northern parts of Scandinavia living apart because of cultural and language barriers, and more or less randomly defined borders.

\section{ACKNOWLEDGEMENT}

We wish to thank Jorunn for doing the translating work.

\section{REFERENCES}

1. Treagust, D. F., Waldrip, B. G. and Horley, J. R. (1993) Effectiveness of ISDN video-conferencing: A case study of two campuses and two different courses. Distance Education, 14 (2).

2. Kristiansen, T. (1993) Five years of research into the use of telecommunications in distance education. Kjeller, Norwegian Telecom Research. (TF-report 29/93).

3. Donath, R. (1993) Intercultural learning with the AT\&T learning network. In: Proceedings of the IFIP TC3 Third Teleteaching Conference. Davies, G. (ed.) IFIP A-29, North Holland, pp. 201-209.

4. Gammon, D and Wilhelmsen, S. (1994) Clinical use of telecommuncations in mental health care. (To be published in Norsk Legejournal, 94). 